\title{
Sharp inequalities related to the constant $e$
}

Yue $\mathrm{Hu}^{1 *}$ and Cristinel Mortici ${ }^{2,3}$

*Correspondence: huu3y3@163.com

${ }^{1}$ College of Mathematics and Informatics, Henan Polytechnic University, Jiaozuo, 454003, China Full list of author information is available at the end of the article

\begin{abstract}
The aim of this work is to extend the results obtained by Batir and Cancan in (Int. J. Math. Educ. Sci. Technol. 40(8):1101-1109, 2009).
\end{abstract}

MSC: Primary 26A09; secondary 33B10; 26D99

Keywords: constant $e_{\text {; inequalities; approximations }}$

\section{Introduction and preliminary results}

Batir and Cancan [1, Theorem 2.5] presented the following sharp inequalities:

$$
\exp \left(1-\frac{n}{2(n+c)^{2}}\right) \leq\left(1+\frac{1}{n}\right)^{n}<\exp \left(1-\frac{n}{2(n+d)^{2}}\right), \quad n \geq 1,
$$

where $c=\frac{1}{\sqrt{2-\ln 4}}-1=0.27649 \cdots$ and $d=\frac{1}{3}$. The proof is based on the fact that the function

$$
\omega(x)=\frac{1}{\sqrt{2\left(\frac{1}{x}-\ln \left(1+\frac{1}{x}\right)\right)}}-x
$$

is strictly increasing on $(0, \infty)$, while inequalities (1) follow from $\omega(1) \leq \omega(n)<\omega(\infty)$. Such an approach of the problem does not offer good results in the left-hand side inequality (1), when $n$ approaches infinity. As we wish to see (1) as an accurate approximation of the form

$$
\left(1+\frac{1}{n}\right)^{n} \approx \exp \left(1-\frac{n}{2(n+\delta(n))^{2}}\right)
$$

we are interested in finding $\delta(n)$ which gives the best such approximation for large values of $n$. Moreover, numerical computations show us that for large values of $n$, the expression $(1+1 / n)^{n}$ gets closer to the right-hand side of (1). This fact suggests us that the best approximation (2) is obtained when $\delta(n)$ tends to $1 / 3$, as $n \rightarrow \infty$. For $\delta(n)=1 / 3$, we deduce

$$
\left(1+\frac{1}{n}\right)^{n} \approx \exp \left(1-\frac{n}{2\left(n+\frac{1}{3}\right)^{2}}\right)
$$

but a better result is

$$
\left(1+\frac{1}{n}\right)^{n} \approx \exp \left(1-\frac{n}{2\left(n+\frac{1}{3}-\frac{1}{12 n}\right)^{2}}\right)
$$

๑) $2014 \mathrm{Hu}$ and Mortici; licensee Springer. This is an Open Access article distributed under the terms of the Creative Commons Attribution License (http://creativecommons.org/licenses/by/2.0), which permits unrestricted use, distribution, and reproduction in any medium, provided the original work is properly cited. 
The rigorous argument is the following theorem, which is also an improvement of the Batir and Cancan inequality (1).

Theorem 1 For every real number $x \geq 1$, we have

$$
\exp \left(1-\frac{x}{2\left(x+\frac{1}{3}-\frac{1}{12 x}\right)^{2}}\right)<\left(1+\frac{1}{x}\right)^{x}<\exp \left(1-\frac{x}{2\left(x+\frac{1}{3}-\frac{1}{12 x}+\frac{23}{540 x^{2}}\right)^{2}}\right) .
$$

Proof Let

$$
f(x)=1-\frac{x}{2\left(x+\frac{1}{3}-\frac{1}{12 x}\right)^{2}}-x \ln \left(1+\frac{1}{x}\right)
$$

and

$$
g(x)=1-\frac{x}{2\left(x+\frac{1}{3}-\frac{1}{12 x}+\frac{23}{540 x^{2}}\right)^{2}}-x \ln \left(1+\frac{1}{x}\right) .
$$

We have $f(1)=\frac{17}{25}-\ln 2=-0.013 \cdots<0, g(1)=\frac{85,351}{121,801}-\ln 2=0.0075 \cdots>0$ and

$$
\begin{aligned}
& f^{\prime \prime}(x)=-\frac{16 x+384 x^{2}+544 x^{3}+15,440 x^{4}+17,664 x^{5}-1}{x(x+1)^{2}(2 x+1)^{4}(6 x-1)^{4}}, \\
& g^{\prime \prime}(x)=\frac{A(x-1)}{x(x+1)^{2}\left(180 x^{2}-45 x+540 x^{3}+23\right)^{4}},
\end{aligned}
$$

where

$$
\begin{aligned}
A(x)= & 1,031,414,482,080 x+3,366,173,125,800 x^{2} \\
& +6,243,358,941,720 x^{3}+7,196,770,443,825 x^{4} \\
& +5,277,779,208,000 x^{5}+2,403,240,062,400 x^{6} \\
& +620,618,112,000 x^{7}+69,480,990,000 x^{8}+137,506,401,616 .
\end{aligned}
$$

Evidently, $f$ is concave, $g$ is convex on $[1, \infty)$, with $f(\infty)=g(\infty)=0$, so $f<0$ and $g>0$ on $[1, \infty)$. The proof is completed.

The same remarks we make on Batir and Cancan's assertion [1, Theorem 2.6], which is proven to have some computation errors, since the expression $(1+1 / n)^{n+1}$ can be approximated for large values of $n$ as

$$
\left(1+\frac{1}{n}\right)^{n+1} \approx \exp \left(1+\frac{n}{2\left(n+\frac{1}{6}\right)^{2}}\right)
$$

but a better result is

$$
\left(1+\frac{1}{n}\right)^{n+1} \approx \exp \left(1+\frac{n}{2\left(n+\frac{1}{6}-\frac{1}{24 n}\right)^{2}}\right)
$$

as we can see from the following. 
Theorem 2 For every real number $x \geq 1$, we have

$$
\exp \left(1+\frac{x}{2\left(x+\frac{1}{6}-\frac{1}{24 x}+\frac{43}{2,160 x^{2}}\right)^{2}}\right)<\left(1+\frac{1}{x}\right)^{x+1}<\exp \left(1+\frac{x}{2\left(x+\frac{1}{6}-\frac{1}{24 x}\right)^{2}}\right)
$$

Proof Let

$$
\begin{aligned}
& u(x)=1+\frac{x}{2\left(x+\frac{1}{6}-\frac{1}{24 x}\right)^{2}}-(x+1) \ln \left(1+\frac{1}{x}\right), \\
& v(x)=1+\frac{x}{2\left(x+\frac{1}{6}-\frac{1}{24 x}+\frac{43}{2,160 x^{2}}\right)^{2}}-(x+1) \ln \left(1+\frac{1}{x}\right) .
\end{aligned}
$$

We have $u(1)=\frac{113}{81}-2 \ln 2=0.00876 \cdots>0, v(1)=\frac{8,448,529}{6,115,729}-2 \ln 2=-0.00485 \cdots<0$ and

$$
\begin{aligned}
& u^{\prime \prime}(x)=\frac{16 x+832 x^{3}+2,624 x^{4}+132,096 x^{5}-1}{x^{2}(x+1)\left(4 x+24 x^{2}-1\right)^{4}}, \\
& v^{\prime \prime}(x)=-\frac{B(x-1)}{x^{2}(x+1)\left(360 x^{2}-90 x+2,160 x^{3}+43\right)^{4}},
\end{aligned}
$$

where

$$
\begin{aligned}
B(x)= & 63,717,283,707,480 x+222,339,093,374,520 x^{2} \\
& +443,153,651,482,080 x^{3}+551,897,650,861,200 x^{4} \\
& +439,841,844,403,200 x^{5}+219,097,061,222,400 x^{6} \\
& +62,377,579,008,000 x^{7}+7,772,423,040,000 x^{8}+7,984,133,521,441 .
\end{aligned}
$$

Evidently, $v$ is concave, $u$ is convex, with $u(\infty)=v(\infty)=0$, so $v<0$ and $u>0$ on $[1, \infty)$. The proof is completed.

\section{Some extensions}

In this section we discuss the problem of approximating $(1+1 / n)^{n+a}, a \in[0,1]$, in the form

$$
\left(1+\frac{1}{n}\right)^{n+a} \approx \exp \left(1-\frac{n}{p(n+q)^{2}}\right), \quad p, q \in \mathbb{R}
$$

More precisely, we propose as an open problem the following approximation formula:

$$
\left(1+\frac{1}{n}\right)^{n+a} \approx \exp \left(1-\frac{\left(\frac{1}{2}-a\right) n}{\left(n+\frac{3 a-2}{6(2 a-1)}\right)^{2}}\right), \quad a \in[0,1] \backslash\left\{\frac{1}{2}\right\} .
$$

Particular values $a=0$ and $a=1$ in (5) lead again to (3) and (4).

The special case $a=1 / 2$ is treated at the final part of this section.

By now, we proved the approximation formula (5) for

$$
a \in\left[0, \frac{4-\sqrt{2}}{7}\right) \cup\left(\frac{1}{2}, \frac{4+\sqrt{2}}{7}\right) \cup(\theta, 1]
$$

where $\theta=0.82462 \cdots$ is the unique real root of $558 a-1,098 a^{2}+675 a^{3}-92$. 
This assertion is sustained by the following three theorems.

Theorem 3 Let $a \in\left[0, \frac{4-\sqrt{2}}{7}\right.$ ). Then there exists $x_{1}>0$ (depending on a) such that for every real number $x \geq x_{1}$, the following inequalities hold true:

$$
\exp \left(1-\frac{\left(\frac{1}{2}-a\right) x}{\left(x+\frac{3 a-2}{6(2 a-1)}-\frac{7 a^{2}-8 a+2}{24(2 a-1)^{2} x}\right)^{2}}\right)<\left(1+\frac{1}{x}\right)^{x+a}<\exp \left(1-\frac{\left(\frac{1}{2}-a\right) x}{\left(x+\frac{3 a-2}{6(2 a-1)}\right)^{2}}\right)
$$

Theorem 4 Let $a \in\left(\frac{1}{2}, \frac{4+\sqrt{2}}{7}\right)$. Then there exists $x_{2}>0$ (depending on a) such that, for every real number $x \geq x_{2}$, the following inequalities hold true:

$$
\exp \left(1+\frac{\left(a-\frac{1}{2}\right) x}{\left(x+\frac{3 a-2}{6(2 a-1)}+\frac{8 a-7 a^{2}-2}{24(2 a-1)^{2} x}\right)^{2}}\right)<\left(1+\frac{1}{x}\right)^{x+a}<\exp \left(1+\frac{\left(a-\frac{1}{2}\right) x}{\left(x+\frac{3 a-2}{6(2 a-1)}\right)^{2}}\right)
$$

Theorem 5 Let $a \in(\theta, 1]$. Then there exists $x_{3}>0$ (depending on a) such that, for every real number $x \geq x_{3}$, the following inequalities hold true:

$$
\exp \left(1+\frac{\left(a-\frac{1}{2}\right) x}{\left(x+\frac{3 a-2}{6(2 a-1)}\right)^{2}}\right)<\left(1+\frac{1}{x}\right)^{x+a}<\exp \left(1+\frac{\left(a-\frac{1}{2}\right) x}{\left(x+\frac{3 a-2}{6(2 a-1)}-\frac{7 a^{2}-8 a+2}{24(2 a-1)^{2} x}\right)^{2}}\right)
$$

Inequalities (6)-(8) are closely related to the functions

$$
s(x)=1+\frac{\left(a-\frac{1}{2}\right) x}{\left(x+\frac{3 a-2}{6(2 a-1)}\right)^{2}}-(x+a) \ln \left(1+\frac{1}{x}\right)
$$

and

$$
t(x)=1+\frac{\left(a-\frac{1}{2}\right) x}{\left(x+\frac{3 a-2}{6(2 a-1)}-\frac{7 a^{2}-8 a+2}{24(2 a-1)^{2} x}\right)^{2}}-(x+a) \ln \left(1+\frac{1}{x}\right) .
$$

We have

$$
s^{\prime \prime}(x)=-\frac{P(x)}{x^{2}(x+1)^{2}(3 a-6 x+12 a x-2)^{4}},
$$

where

$$
\begin{aligned}
P(x)= & 648\left(7 a^{2}-8 a+2\right)(2 a-1)^{3} x^{3} \\
& +48(18 a-11)(3 a-2)(3 a-1)(2 a-1)^{2} x^{2} \\
& +(27 a-2)(2 a-1)(3 a-2)^{3} x \\
& +a(3 a-2)^{4}
\end{aligned}
$$

and

$$
t^{\prime \prime}(x)=\frac{Q(x)}{x^{2}(x+1)^{2}\left(24(2 a-1)^{2} x^{2}+4(2 a-1)(3 a-2) x-\left(7 a^{2}-8 a+2\right)\right)^{4}},
$$


where

$$
\begin{aligned}
Q(x)= & 3,072\left(558 a-1,098 a^{2}+675 a^{3}-92\right)(2 a-1)^{6} x^{6} \\
& +64\left(94,140 a^{2}-31,392 a-122,256 a^{3}+57,753 a^{4}+3,860\right)(2 a-1)^{5} x^{5} \\
& +128\left(816 a-3,556 a^{2}+7,164 a^{3}-6,813 a^{4}+2,484 a^{5}-68\right)(2 a-1)^{4} x^{4} \\
& +64\left(7 a^{2}-8 a+2\right)\left(942 a^{2}-356 a-1,086 a^{3}+465 a^{4}+48\right)(2 a-1)^{3} x^{3} \\
& +16\left(10 a-14 a^{2}+9 a^{3}-4\right)\left(7 a^{2}-8 a+2\right)^{2}(2 a-1)^{2} x^{2} \\
& +\left(41 a^{2}-24 a-2\right)\left(7 a^{2}-8 a+2\right)^{3}(2 a-1) x \\
& -a\left(7 a^{2}-8 a+2\right)^{4} .
\end{aligned}
$$

Proofs of Theorems 3 and 4 For $a \in\left[0, \frac{4-\sqrt{2}}{7}\right) \cup\left(\frac{1}{2}, \frac{4+\sqrt{2}}{7}\right)$, the leading coefficients of the polynomials $P$ and $Q$ are negative. We are in a position to consider $j>0$ (depending on $a$ ) such that $P(x)<0$ and $Q(x)<0$, for every $x \in[j, \infty)$. By (9) and (10), $s$ is concave and $t$ is convex. But $s(\infty)=t(\infty)=0$, so $s<0$ and $t>0$ on $[j, \infty)$.

Now inequalities $s(x)<0$ and $t(x)>0$, for every $x \in[j, \infty)$ are (6) and (7) and we are done.

Proof of Theorem 5 For $a \in(\theta, 1]$, the leading coefficients of polynomials $P$ and $Q$ are positive. We are in a position to consider $l>0$ (depending on $a$ ) such that $P(x)>0$ and $Q(x)>0$, for every $x \in[l, \infty)$. By (9) and (10), now $s$ is convex and $t$ is concave. But $s(\infty)=$ $t(\infty)=0$, so $s>0$ and $t<0$ on $[l, \infty)$.

Now inequalities $s(x)>0$ and $t(x)<0$, for every $x \in[l, \infty)$ are (6) and (7) and we are done.

The special case $a=1 / 2$ provides the approximation formula

$$
\left(1+\frac{1}{n}\right)^{n+\frac{1}{2}} \approx \exp \left(1+\frac{n}{12\left(n+\frac{1}{3}\right)^{3}}\right) \text {. }
$$

It is sustained by the following.

Theorem 6 For every real number $x \geq 1$, the following inequalities hold:

$$
\exp \left(1+\frac{x}{12\left(x+\frac{1}{3}\right)^{3}}\right)<\left(1+\frac{1}{x}\right)^{x+\frac{1}{2}}<\exp \left(1+\frac{x}{12\left(x+\frac{1}{3}-\frac{7}{90 x}\right)^{3}}\right)
$$

Proof Let us define the functions

$$
b(x)=1+\frac{x}{12\left(x+\frac{1}{3}\right)^{3}}-\left(x+\frac{1}{2}\right) \ln \left(1+\frac{1}{x}\right)
$$

and

$$
c(x)=1+\frac{x}{12\left(x+\frac{1}{3}-\frac{7}{90 x}\right)^{3}}-\left(x+\frac{1}{2}\right) \ln \left(1+\frac{1}{x}\right) .
$$


We have

$$
b^{\prime \prime}(x)=-\frac{15 x+171 x^{2}+189 x^{3}+1}{2 x^{2}(x+1)^{2}(3 x+1)^{5}}
$$

and

$$
c^{\prime \prime}(x)=\frac{R(x-1)}{2 x^{2}(x+1)^{2}\left(30 x+90 x^{2}-7\right)^{5}},
$$

where

$$
\begin{aligned}
R(x)= & 44,117,710,950 x+122,222,582,550 x^{2}+187,066,692,000 x^{3} \\
& +170,643,105,000 x^{4}+92,646,666,000 x^{5}+27,671,382,000 x^{6} \\
& +3,499,200,000 x^{7}+6,793,216,207 .
\end{aligned}
$$

Evidently, $b$ is concave, $c$ is convex, with $b(\infty)=c(\infty)=0$, so $b<0$ and $c>0$ on $[1, \infty)$. The proof is completed.

In case $a=1 / 2$, the entire asymptotic representation

$$
\left(1+\frac{1}{x}\right)^{x+1 / 2} \sim \exp \left(1+\frac{\frac{1}{12} x}{\left(x+\sum_{j=0}^{\infty} \frac{a_{j}}{x^{j}}\right)^{3}}\right), \quad x \rightarrow \infty,
$$

can be constructed. In this sense, we write (12) as

$$
(f(x))^{-1 / 3} \sim x+\sum_{j=0}^{\infty} \frac{a_{j}}{x j}
$$

where

$$
f(x)=\frac{\left(x+\frac{1}{2}\right) \ln \left(1+\frac{1}{x}\right)-1}{\frac{1}{12} x} .
$$

By using the Maclaurin expansion of $\ln (1+t)$, with $t=x^{-1}$, we deduce that

$$
f(x)=\sum_{j=3}^{\infty}(-1)^{j-1} \frac{6(j-2)}{j(j-1) x^{j}}
$$

Now the coefficients $a_{j}$ in (12) can be inductively obtained by equating the following relation:

$$
\left\{\sum_{j=3}^{\infty}(-1)^{j-1} \frac{6(j-2)}{j(j-1) x^{j}}\right\} \times\left\{x+\sum_{j=0}^{\infty} \frac{a_{j}}{x^{j}}\right\}^{3}=1 .
$$

The first coefficients are $a_{0}=\frac{1}{3}, a_{1}=-\frac{7}{90}$ (see (11)), then $a_{2}=\frac{16}{405}, a_{3}=-\frac{2,141}{85,050}, \ldots$.

Further research in the problem of approximating the constant $e$ can be found in [1-4]. Finally, we leave as an open problem the approximation formula (5) for values of $a \in[0,1]$ other than those discussed in this paper. 


\section{Competing interests}

The authors declare that they have no competing interests.

\section{Authors' contributions}

The paper is a joint work of all the authors who contributed equally to the final version of the paper. All authors read and approved the final manuscript.

\section{Author details}

${ }^{1}$ College of Mathematics and Informatics, Henan Polytechnic University, Jiaozuo, 454003, China. ${ }^{2}$ Valahia University of Târgovişte, Bd. Unirii 18, Târgovişte, 130082, Romania. ${ }^{3}$ Academy of Romanian Scientists, Splaiul Independenţei 54, Bucharest, 050094, Romania.

\section{Acknowledgements}

The authors would like to thank the Editor and the referees for the comments that improved the initial form of this manuscript. The work of Cristinel Mortici was supported by a grant of the Romanian National Authority for Scientific Research, CNCS-UEFISCDI project number PN-II-ID-PCE-2011-3-0087. Cristinel Mortici contributed to this work during his visit at National Technical University of Athens, Greece. He would like to thank Prof. Themistocles M Rassias for hospitality.

Received: 8 September 2014 Accepted: 25 September 2014 Published: 3 October 2014

\section{References}

1. Batir, N, Cancan, M: Sharp inequalities involving the constant $e$ and the sequence $(1+1 / n)^{n}$. Int. J. Math. Educ. Sci. Technol. 40(8), 1101-1109 (2009)

2. Agarwal, RP, Sen, SK: Pi, Epsilon, Phi with MATLAB: Random and Rational Sequences with Scope in Supercomputing Era. Cambridge Scientific Publishers, Cambridge (2011)

3. Mortici, C: Refinements of some bounds related to the constant e. Miskolc Math. Notes 12(1), 105-111 (2011)

4. Mortici, C: A quicker convergence toward the gamma constant with the logarithm term involving the constant $e$. Carpath. J. Math. 26(1), 86-91 (2010)

doi:10.1186/1029-242X-2014-382

Cite this article as: Hu and Mortici: Sharp inequalities related to the constant e. Journal of Inequalities and Applications 2014 2014:382.

\section{Submit your manuscript to a SpringerOpen ${ }^{\ominus}$ journal and benefit from:}

- Convenient online submission

- Rigorous peer review

- Immediate publication on acceptance

- Open access: articles freely available online

- High visibility within the field

- Retaining the copyright to your article 